\title{
A New Computed Torque Control System with an Uncertain RBF Neural Network Controller for a 7-DOF Robot
}

\author{
Liandong WANG* , Xiaogin ZHOU, Tiehua HU
}

\begin{abstract}
A novel percutaneous puncture robot system is proposed in the paper. Increasing the surgical equipment precision to reduce the patient's pain and the doctor's operation difficulty to treat smaller tumors can increase the success rate of surgery. To attain this goal, an optimized Computed Torque Law (CTL) using a radial basis function (RBF) neural network controller (RCTL) is proposed to improve the direction and position accuracy. BRF neural network with an uncertain term (URBF) which is able to compensate the system error caused by the imprecision of the model is added in the RCTL system. At first, a 7-DOF robotic system is established. It consists of robotic arm and actuator control channels. Now, the RBF compensator is added to the CTL to adjust the robot arm to reduce the position and direction errors. The angle and velocity errors of the robot arm are compensated using the RBF controller. According to the Lyapunov theory, the accuracy of torque control system depends on path tracking errors, inertia of robot, dynamic parameters and disturbance of each joint. Compared to general CTL approaches, the precision of a 7-DOF robot could be improved by adjusting the RBF parameters.
\end{abstract}

Keywords: compensating controller; computed torque; control system; RBF neural network; robot control

\section{INTRODUCTION}

The interventional radiology is based on the percutaneous puncture technique. The robot used in puncture surgery is also a new type of medical equipment. Now, the image processing and robot automatic control technologies are integrated with the technology of puncture surgery robot to improve the accuracy and performance of the puncture surgery.

The operation follow-up of a CT image navigating robot as one type of these surgical robots is given as follows: At first, the image data of the lesion area is collected and reconstructed by a CT equipment [29]. Now, three-dimensional image data could be employed by a doctor to choose a needle entry and a lesion point. After confirming these points, their coordinate information is transmitted to the surgical robot system. This information is converted by the robot system into the coordinate information of the robot. Then, the robot system moves the needle to the specified position and direction. Finally, when the doctor confirms that the robot is in place, the puncture process is performed. The main shortcomings of the technical scheme of this process are:

1. Nowadays, all surgical robots are positioning robots. These robots can help doctors to place the needle only in a planned position and direction. This means that automatic needling movement could not be realized through them.

2. Since it is difficult to construct a precise robotic model due to some factors such as mechanical damping and inertia in the motion process, the accuracy could be degraded during a robot moving.

3. At the same time, it is inconvenient for the present robot to place the needle twice. By using the automatic needle insertion robot system, the needle insertion direction could be adjusted at all times.

The robot study is always focusing on nonlinear and high precision controlling strategies. However, due to mechanical errors, system damping and external interference, employing these strategies is usually difficult. Therefore, to compensate the effects of nonlinearity and disturbance, a superior control strategy should be employed [1, 2]. As a result, higher accuracy could be obtained for the surgical robots.
A force feedback control system with two loops has been proposed by Zarrouk, Z. [1] where nonlinear state feedback and PID controllers have been utilized in the inner and outer loops, respectively. In [2], a similar approach has been proposed where an Iterative Learning Control (ILC) approach has been employed in the outer loop. A haptic-feedback adaptive controlling system has been studied in [3]. Another novel cable-driven 6-DOF serial force-reflecting haptic device has been presented for a robotic minimally invasive surgery (MIS) system [4] by $\mathrm{Qu}$ et al. A work space has been analyzed beyond above scope in system [5]. An adaptive controlling system for an uncertain kinematic and dynamic robotic manipulator has been proposed by Hanlei Wang [6]. A special Machine Learning Algorithms (MLA) case has been found in the positional learning algorithm controller for MIS by Bruno, D. et al. [16]. To achieve high-precision position tracking, an adaptive trajectory tracking neural network controller with RBF has been constructed for an n-link robot manipulator with a robust compensator [7]. An artificial neural network (ANN) control system with an adaptive nonlinear controller has been put forward for a robot arm [8]. A fuzzy sliding mode control system with a RBF neural network (RBFNN) has been proposed for trajectory tracking of a robot manipulator [9]. A new artificial consciousness-planning controller has been utilized to solve the robot trajectory-planning problem [10]. Once, neural network control systems have been developed to compensate for the systemic nonlinearity by employing multi-level learning stages [11-13]. Such approaches like the collision detection and reaction make the controller design process difficult especially in the presence of dynamic collisions $[14,15]$. A novel model predictive control (MPC) based master-slave force hybrid coordinated motion control system has been established $[16,17]$. Two model-free sliding mode control system (MFSMCS) structures have been constructed [18]. Although these control structures are identical to modelfree intelligent proportional integral (IPI) controlling system, their superiorities have been demonstrated through experimental tests [18]. A fuzzy logic controller (FLC) has been employed to construct a trajectory tracking control system for a wheeled mobile robot [20]. In a trajectory tracking control system, the feed forward velocity data was 
added to the control system as the correcting data from the FLC in accordance with the postures errors. In [21], a comparison between fuzzy proportional derivative control (PD) and sliding mode control (SMC) models for controlling a 2-DOF robot manipulator has been performed.

In this paper, a 7-DOF robotics manipulator is described.

In this paper, an automatic needle insertion robot is proposed, which cannot only locate an operation position automatically, but also insert a needle automatically. Doctors only need to monitor the needle insertion status at all times. Then, an optimizing CTL control system which is called as RCTL based on this percutaneous puncture robot is presented and simulated. From simulation analysis, the performance and the accuracy of RCTL control system is suitable for this kind of percutaneous puncture.

In section 2, a system composition of the robot is introduced. In section 3, the mechanical dimensions of a robot arm and a linear puncture actuator are described. In section 4 , an operation process of the puncture surgery robot is described and the general CTL control system is illustrated. In section 5, the uncertain RBF (URBF) neuralnetwork structure is used to improve the control precision of the robot system in the RCTL. In section 6, the simulation analysis is carried out based on the robotic system. At last, we summarize the performance and feasibility of the surgical robot.

\section{AUTOMATIC NEEDLE INSERTION ROBOT FOR THE PERCUTANEOUS PUNCTURE}

A new teleoperation medical robotic system is presented in this paper where its structure is shown in Fig. 1. This structure involves an automatic needle inserting system with an on-line monitoring capability.
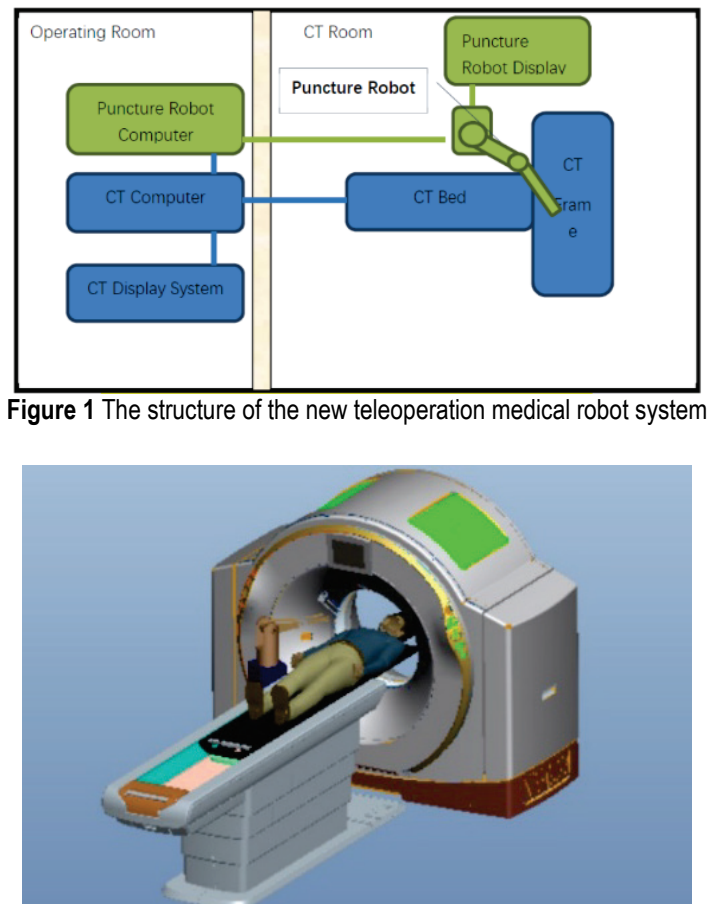

Figure 2 The robotic model for the new teleoperation medical robot system

The working mode of this robot system is shown in Fig. 2. Fig. 3 shows a puncture robot arm system where its puncture actuator is shown in Fig. 4.

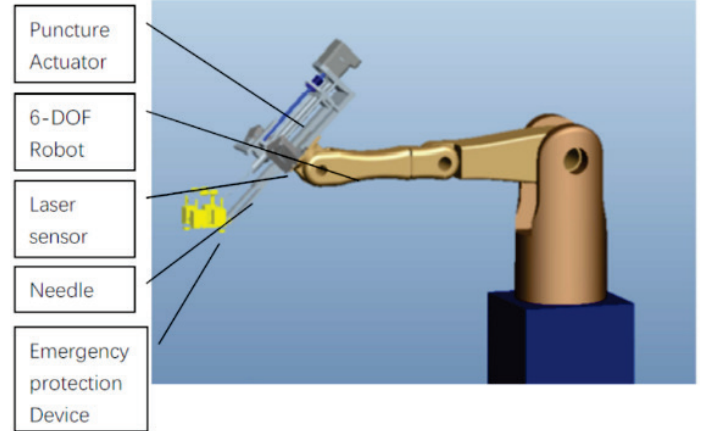

Figure 3 The automatic needle inserting actuator with a robot arm for the percutaneous puncture surgery

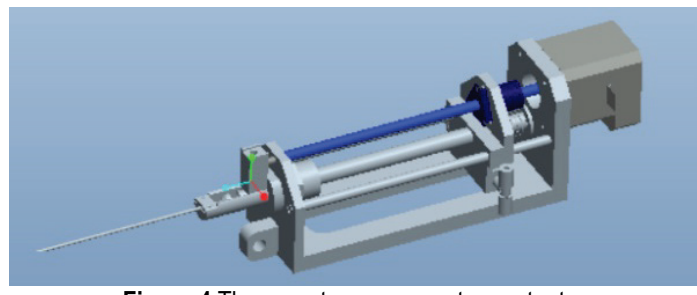

Figure 4 The percutaneous puncture actuator

\section{CONFIGURATION AND MATHEMATICAL MODEL}

The robot structure is shown in Fig. 3. As could be seen from Fig. 3, the robot consists of two automatic parts. Controlling the needle direction at the entry point and approaching the needle insertion point are performed by the first part which is a 6-DOF robot arm.
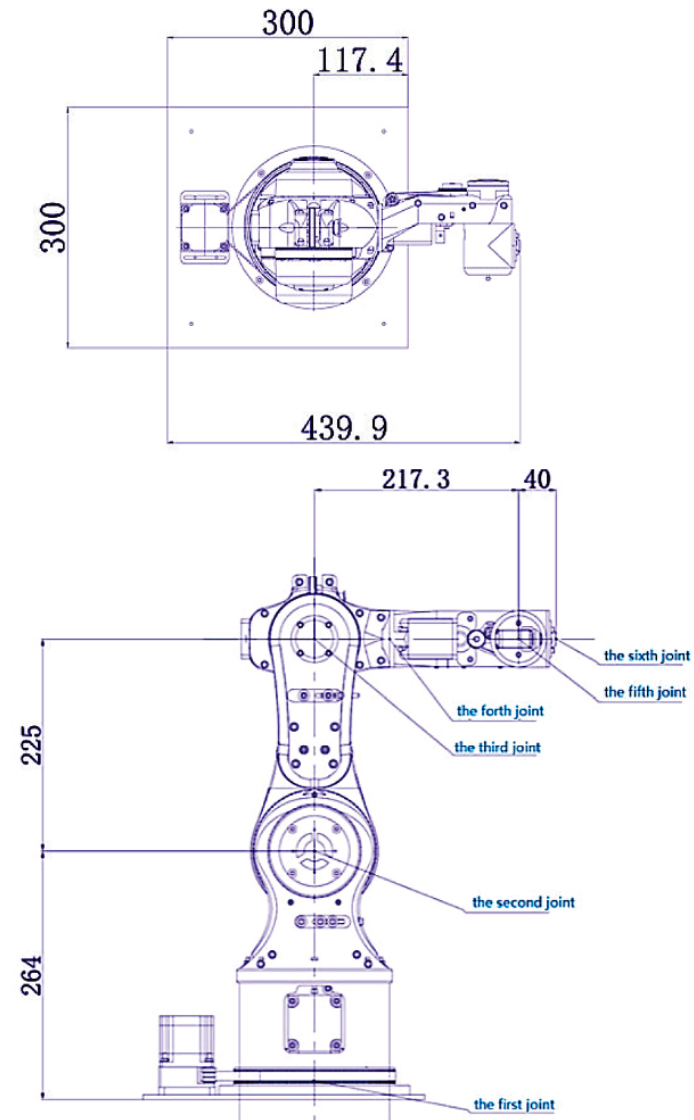

Figure 5 The dimensions of the robotic arm (unit: $\mathrm{mm}$ )

The physical dimension of the robotic arm is shown in Fig. 5. The second part which is a 1-DOF linear actuator 
assembly is employed to insert needle automatically and control the depth and the speed of the needle. The dimension and structure of the actuator assembly which is removal of laser sensors and the emergency protection device is shown in Fig. 6.

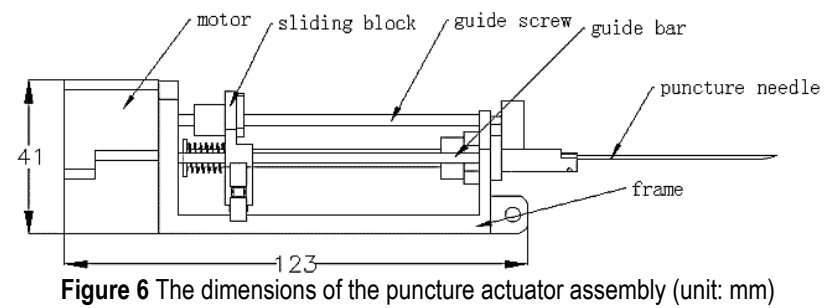

\section{THE PERCUTANEOUS PUNCTURE ROBOT CONTROL SYSTEM}

Various control methods have been presented for different surgical robotic systems in the literature. Neural Network Control (NNC), Force Feedback, Position Feedback (PF), MPC, traditional PID controller, and Repetitive Predictive Control (RPC) are some of these methods.

The propositional robotic system's workflow is shown in Fig. 7. The system is composed of electrical, control, and mechanical subsystems. The robot control process could be disturbed by several nonlinear factors such as electrical parameters fluctuation, friction damping, robot deformation, and machining errors. Therefore, it is difficult to adjust the motion parameters of the robot due to the above problems. Thus, this paper focuses on the robotic controlling system.

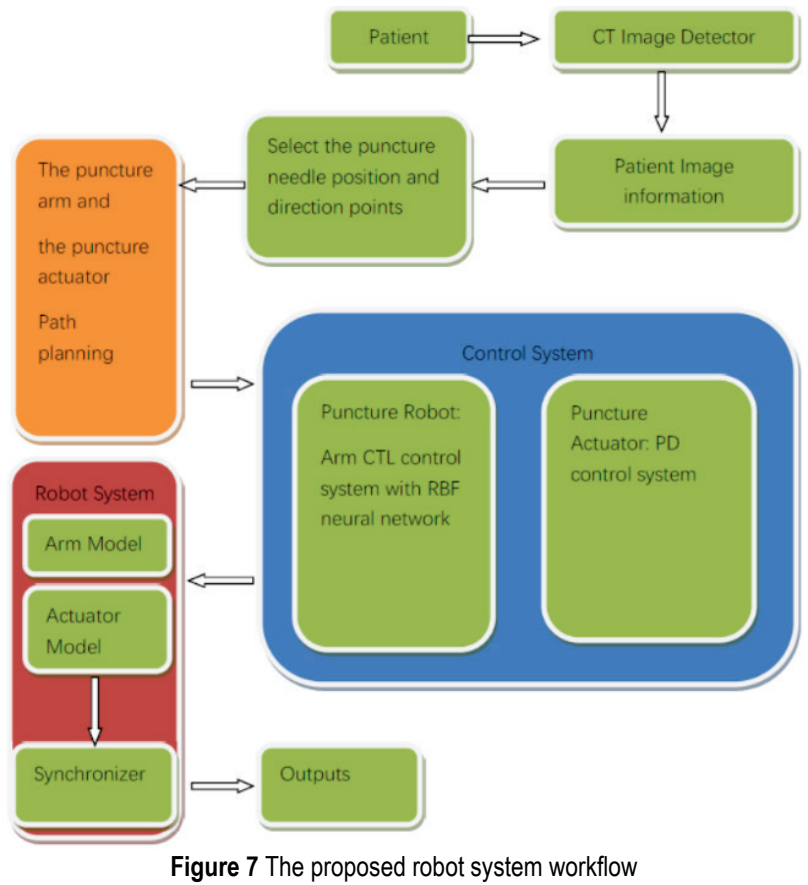

To develop new robotic systems, the following requirements should be considered in the design procedure:

Firstly, it is important to know that mechanical and structural characteristics of the percutaneous puncture robot arm and the puncture actuator could affect the surgical robot control system designing during the robot system development.

Secondly, the needle position should be accurately measured by using a magnetic positional feedback, binocular vision camera collecting, or laser range radar sensor and so on.

Thirdly, on-line measurement of the robot position is necessary for trajectory planning and tracking.

A second-order nonlinear differential equation is employed to describe the dynamic performance for an $N$ joints robot as follows [8]:

$$
D(q) \ddot{q}+C(q, \dot{q})+G(q)=\tau+\tau_{d}
$$

where $q \in R^{n}$ is the Angular displacement of the joint, $D(q) \in R^{n \times n}$ is the inertial matrix of the robot, $C(q, \dot{q}) \in R^{n}$ describes the centrifugal and Coriolis forces, $G(q) \in R^{n}$ denotes the gravitational term, $\tau \in R^{n}$ is the control torque and $\tau_{d} \in R^{n}$ describes the errors and disturbances.

Since the puncture actuator could be considered as a 1DOF robot, the PD control algorithm could be applied to this part. Because the puncture actuator is simple and the parameter model is relatively accurate, the PD control system can meet the actuator requirements, completely.

The actuator dynamics could be described as:

$$
D\left(q_{A}\right)_{A} \ddot{q}_{A}+C\left(q_{A}, \dot{q}_{A}\right)_{A}+G\left(q_{A}\right)_{A}=\tau_{A}+\tau_{d A}
$$

where the angular displacement, the inertial matrix, the centrifugal and coriolis forces, the gravitational term, the control torque, and the errors and disturbances of the actuator motor are denoted by $q_{A} \in R^{1}, D\left(q_{A}\right)_{A} \in R^{1 \times 1}$, $C(q, \dot{q}) \in R^{1}, G(q) \in R^{1}, \tau \in R^{1}, \tau_{d} \in R^{1}, \quad$ and $\quad \tau_{d}=0$, respectively.

The CTL block diagram as a kind of forward controlling laws is shown in Fig. 8. According to the forward control system property, the forward control can eliminate the robot system nonlinearities and improve its linear performance and motion accuracy. Thus, the CTL method could be applied to the robot system.

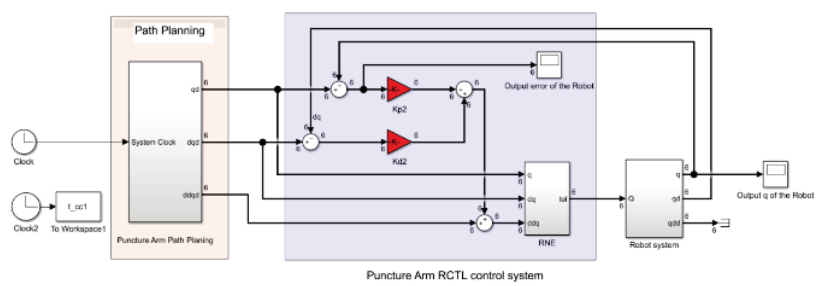

Figure 8 The block diagram of the CTL system

\section{UNCERTAIN RBF NEURAL NETWORK}

RBF is a three-layer feedforward neural network with a single hidden layer where its structure is shown in Fig. 9. The neural network structure of locally adjusted and mutually covered receptive fields (or Receptive Fields) in the human brain could be simulated via the RBF network. It has been proved that arbitrary continuous functions with 
arbitrary accuracy could be approximated by the RBF network. The RBF neural network structure is based on the $\mathrm{BP}$ neural network. In theory, any non-linear function could be approximated via a BP neural network with three or more layers. However, since the BP network is a global approximation network, its weights should be retuned when the samples are learned. Thus, it has a low convergence speed. The mapping process from inputs to outputs is nonlinear, while the mapping process from a hidden layer to an output layer is linear. A local approximation RBF neural network can greatly speed up the learning rate and avoid local minimum. So it makes the RBF suitable for a real-time control system. The RBF network is utilized to construct a neural network based control structure for the robot system, which can effectively improve its accuracy, robustness, and adaptability.

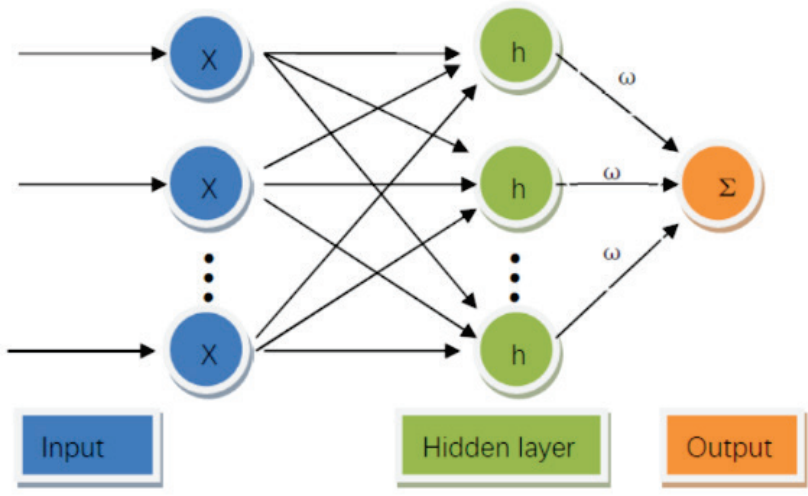

Figure 9 The RBF neural network structure

The learning process of the RBF network is similar to the BP network, while their activation functions are different. The sigmoid function is employed in the hidden layer of the BP network. Since the sigmoid function value is non-zero in the infinite range of the input space, the BP network could be considered as a kind of global approximation neural networks. However, a Gaussian basis function is utilized as the activation function in the RBF network. Since its value is non-zero in the limited range of the input space, this network is called a local approximation neural network.

In the RBF network structure, the following notations are considered:

$\boldsymbol{X}=\left[x_{1}, x_{2}, \cdots, x_{n}\right]^{\mathrm{T}}$, is the input vector of the network
$\boldsymbol{W}=\left[\omega_{1}, \omega_{2}, \cdots, \omega_{m}\right]^{\mathrm{T}}$, is the weight vector of the RBF neural network

$\boldsymbol{H}=\left[h_{1}, h_{2}, \cdots, h_{m}\right]^{\mathrm{T}}$, is the radial basis vector of RBF network where $h_{j}$ is the Gaussian basis function and is calculated as:

$h_{j}=\exp \left(-\frac{\left\|X-c_{j}\right\|^{2}}{2 b_{j}^{2}}\right) j=1,2, \cdots, m$

$\boldsymbol{c}_{j}=\left[c_{j 1}, c_{j 2}, \cdots, c_{j n}\right]$ is the central vector of the $j$-th node in the network. The RBF network output $\left(y_{m}(t)\right)$ could be calculated as:

$$
y_{m}(t)=\sum_{i=1}^{m} \omega_{i} h_{i}
$$

According to the gradient descent method, the output weight tuning, the central vector and the node base width parameters could be updated according to the following iterative algorithm:

$$
\boldsymbol{B}=\left[b_{1}, b_{2}, \cdots, b_{m}\right]^{\mathrm{T}} \text { denotes the base width vector of the }
$$
network where $b_{j}>0$ is the base width parameter for $j$-th node. This parameter could be updated as

$$
\begin{aligned}
& \omega_{j}(t)=\omega_{j}(t-1)+\eta\left(y(t)-y_{m}(t)\right) h_{j}+ \\
& +\alpha\left(\omega_{j}(t-1)-\omega_{j}(t-2)\right) \\
& b_{j}(t)=b_{j}(t-1)+\eta \triangle b_{j}+\alpha\left(b_{j}(t-1)-b_{j}(t-2)\right)
\end{aligned}
$$

where,

$$
\triangle b_{j}(t)=\left(y(t)-y_{m}(t)\right) \omega_{j} h_{j} \frac{\left\|X-c_{j}\right\|^{2}}{2 b_{j}^{2}}
$$

The weight tuning vector of the network is denoted by $\boldsymbol{W}=\left[\omega_{1}, \omega_{2}, \cdots, \omega_{m}\right]^{\mathrm{T}}$.

The central vector could be adapted as

$c_{j i}(t)=c_{j i}(t-1)+\eta \triangle c_{j i}+\alpha\left(c_{j i}(t-1)-c_{j i}(t-2)\right)$

where

$\Delta c_{j i}=b_{j}\left(y(t)-y_{m}(t)\right) \omega_{j} \frac{x_{i}-c_{j i}}{b_{j}^{2}}$

where $\eta$ and $\alpha$ are the learning rate and the momentum factor, respectively.

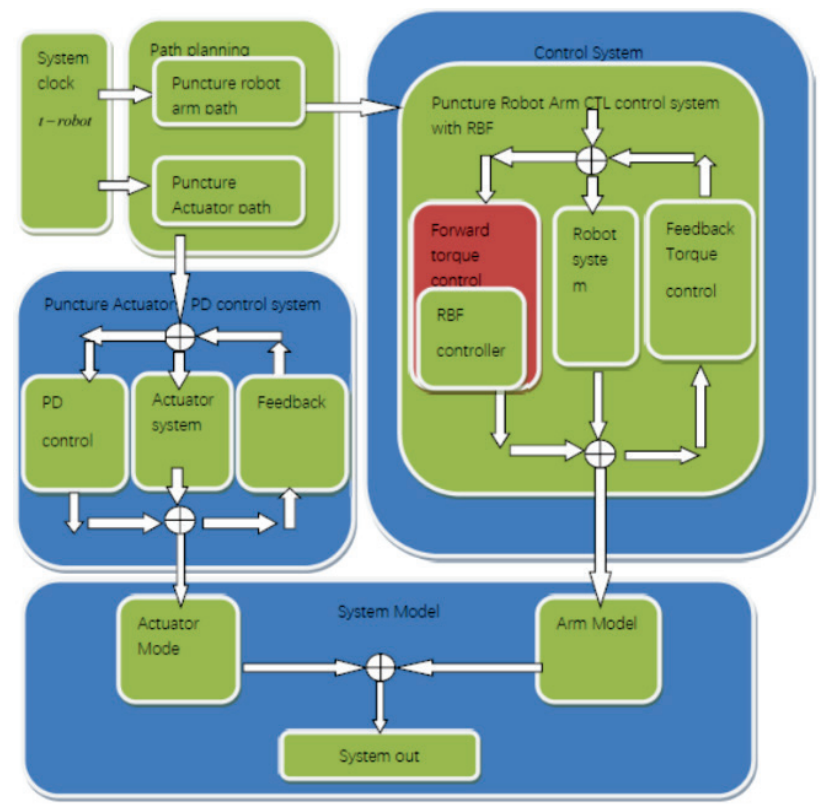

Figure 10 The puncture robot control system 
Since CTL system errors of the robot are uncoupled for joints, and dynamic characteristics of the robot are relatively independent, an RBF compensation controller is added to the CTL control loop to deal with the robot system nonlinearity (see Fig. 10). At last, motions of the robot and the actuator are synchronized with the path planning clock's time step (Fig. 10).

According to Eq. (1), we have

$$
\tau=D(q) \ddot{q}+C(q, \dot{q})+G(q)-\tau_{d}
$$

Consider that the model is accurate, and $\tau_{d}=0$. Now, the control law could be calculated as

$$
\tau=D(q)\left(\ddot{q}_{d}-k_{v} \dot{e}-k_{p} e\right)+C(q, \dot{q})+G(q)
$$

Now, by replacing (11) in (10), the characteristic equation of the stable closed loop system is obtained as:

$$
\ddot{e}+k_{v} \dot{e}+k_{p} e=0
$$

where $q_{d}$ is the expected angular displacement of the joint and the angular displacement error $(e)$ and its first and second derivatives are calculated as

$$
\begin{aligned}
& e=q-q_{d} \\
& \dot{e}=\dot{q}-\dot{q}_{d} \\
& \ddot{e}=\ddot{q}-\ddot{q}_{d}
\end{aligned}
$$

In an actual robot system, it is very difficult to describe an accurate model. Thus, there is an inaccuracy term in the model. It's impossible to obtain exact expressions for $D(q), C(q, \dot{q})$ and $G(q)$. Thus, their nominal terms $D_{0}(q)$, $C_{0}(q, \dot{q})$ and $G_{0}(q)$ are utilized in the design procedure. Or

$$
\begin{aligned}
& \tau_{0}=D_{0}(q) \ddot{q}+C_{0}(q, \dot{q})+G_{0}(q) \\
& \tau_{0}=D_{0}(q)\left(\ddot{q}_{d}-k_{v} \dot{e}-k_{p} e\right)+C_{0}(q, \dot{q})+G_{0}(q)
\end{aligned}
$$

where $\tau_{0}$ is the ideal torque.

According to (11) and (17), the following modeling inaccuracy term is obtained

$$
\begin{aligned}
& \varepsilon(t)=\tau-\tau_{0}= \\
& =\left(D_{0}(q)\left(\ddot{q}_{d}-k_{v} \dot{e}-k_{p} e\right)+C_{0}(q, \dot{q})+G_{0}(q)+\tau_{d}\right)-(18) \\
& -(D(q)+C(q, \dot{q})+G(q)) \\
& \varepsilon(t)=D_{0}^{-1}\left(\Delta D \ddot{q}+\Delta C \dot{q}+\Delta G+\tau_{d}\right) \\
& \triangle D=D_{0}-D, \Delta C=C_{0}-C, \triangle G=G_{0}-G
\end{aligned}
$$

The RBF neural network could be employed to approximate the actual model. This compensation controller can be set as:

$$
\varepsilon(t) \approx y_{m}(t)+\varepsilon_{0}
$$

Here, $y_{m}(t)$ is the unknown function of the neural network and $\varepsilon_{0}$ is the inaccuracy error. It could be proved that if $W$ and $H$ exist for limited number of neurons, the inaccuracy error $\varepsilon_{0}$ becomes approximately zero. In this case, the existence of a limited neuron number constituting the network model supplementary controller can compensate for the system error.

According to the above description, the computed torque control (CTL) system for this puncture robot is described as:

$$
\begin{aligned}
& Q=D_{0}(q)\left\{\ddot{q}_{d}+K_{v}\left(\dot{q}_{d}-\dot{q}\right)+K_{p}\left(q_{d}-q\right)\right\}+ \\
& +C_{0}(q, \dot{q}) \dot{q}+G_{0}(q)+K_{t} \tau_{2}
\end{aligned}
$$

where the expected angular displacement, velocity, and acceleration of the joint are denoted by $q_{d} \in R^{n}$, $\dot{q}_{d} \in R^{n}$, and $\ddot{q}_{d} \in R^{n}$, respectively. $K_{p}$ and $K_{v}$ are the gain matrices of position and speed, respectively. $K_{t}$ is the gain vector of $\tau_{2} . \quad Q \in R^{n}$ is the control torque matrix.

The control law of the URBF network could be calculated as

$$
\begin{aligned}
& \tau_{2}=-D_{0}(q) y_{m}(t) \\
& y_{m}(t)=\boldsymbol{W}^{\mathrm{T}} \boldsymbol{H}
\end{aligned}
$$

Due to differences among the position, orientation, and effects of gravity and Coriolis forces for different joints at different time steps, the CTL method is applied to the system to control the percutaneous puncture robot. $\boldsymbol{W}=\left[\omega_{1}, \omega_{2}, \cdots, \omega_{m}\right]^{\mathrm{T}}$ is the weight tuning vector in the neural network mentioned above.

Then the output acceleration is computed by a RNE module.Thus, the approximation term of the RBF network with uncertain approximation is employed to compensate for the CTL control system of the model. This could be employed as a feedforward compensation controller. The RBF uncertain network approximation term (URBF) is applied to the CTL system to compensate for the control feedback path through $q$.

In the RBF controller, a RBF module is also employed. Once the system starts, it could not be stabilized. The robot system becomes stable after a little period time. The system time, $t_{-} c c 1$, could be adjusted based on the planned path timestep of the puncture robotics controlling system. During this period, the robot system becomes increasingly stable and periodical, and then the robot moves along the planned trajectory. After getting the activating signal from the path-planning step, the robotics controlling system begins to work and the RBF controller starts to work, too. The needle will also move along the planned pathway.

The puncture actuator that is the needle driving system is driven through a simple linear motor.

Due to its high precision, the CTL method is suitable for the robot control system. 


\section{SIMULATION RESULTS}

The CTL control system with the URBF controller is simulated with MATLAB software. The parameters of the puncture actuator of the CTL control system are shown in Tab. 1 while the parameters of the CTL control system
(RCTL) with the URBF network are shown in Tab. 2. The input data is the trajectory planning path matrix. The CTL control system (RCTL) with the URBF controller is shown in Fig. 11.

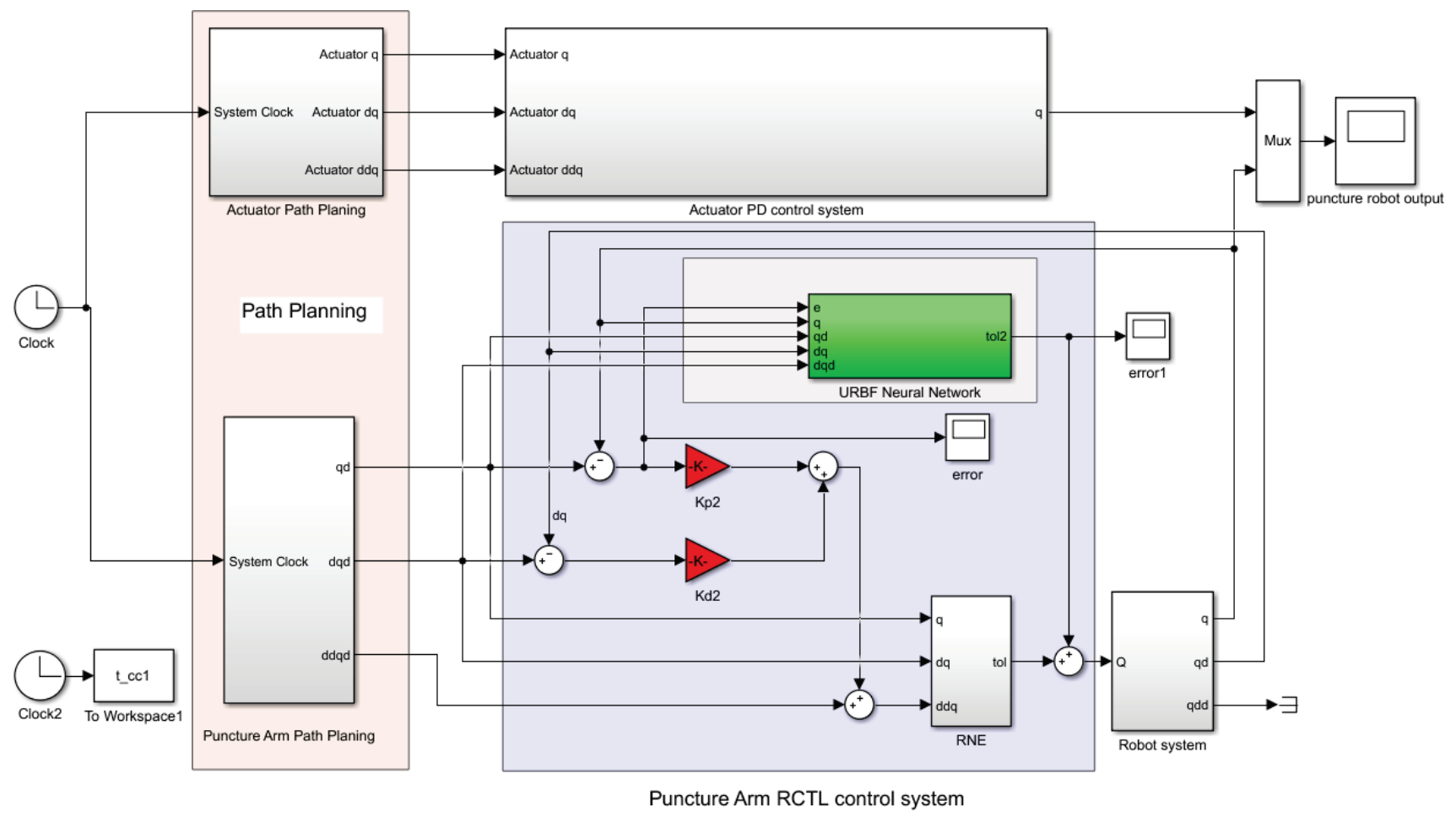

Figure 11 The CTL control system with the RBFcontroller

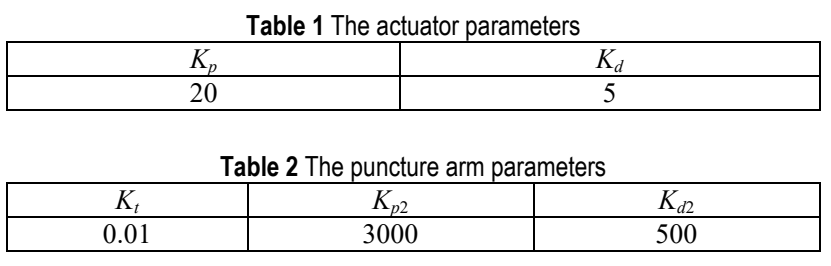

Timer parameter, $t \_c c 1$ as the unique timer is utilized in both control channels including the robotics arm and the above-mentioned actuator. It is considered as $t c c 1=4 \mathrm{~s}$. Now, both control channels are triggered synchronously. The time step length is considered the same for both control channels.

Six joints' errors of the robot are shown in Fig. 12. Two minutes later, the errors approximately become fixed and the robot system becomes stable.

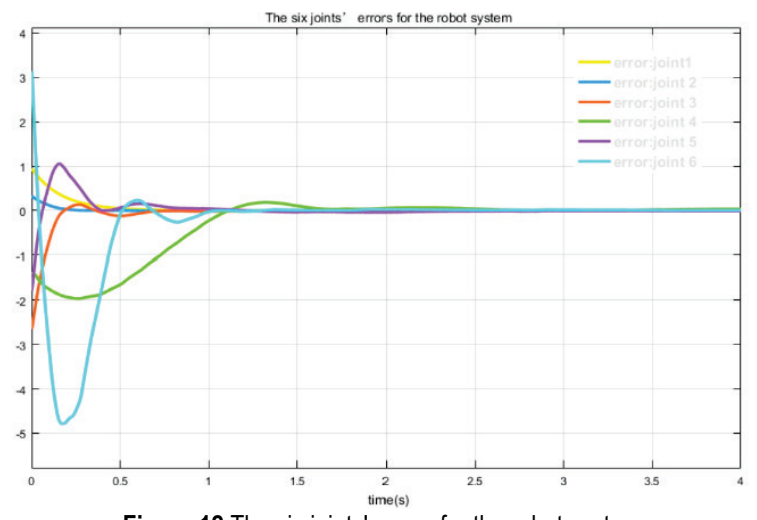

Figure 12 The six joints' errors for the robot system
Fig. 13 shows the actuator performance for the planning path and the positional accuracy of the needle at the final time step (Tab. 5). As could be seen from Tab. 5, the accuracy between the planed path and the control processing path is less than $0.007873 \mathrm{~mm}$.

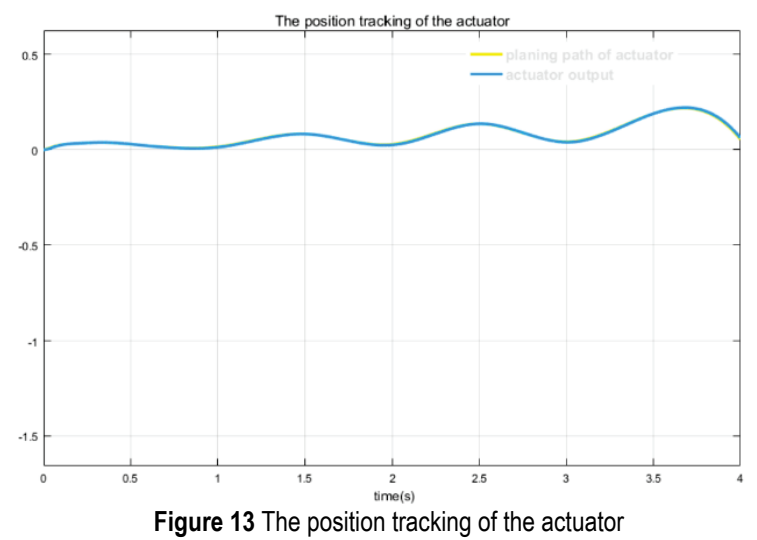

Figs. 14 to 19 show the performance and the position data between the puncture arm planned path and joints in the process. It could be seen form Tab. 5 that the position error between the expected path and the obtained one is less than $0.03216 \mathrm{~mm}$. 


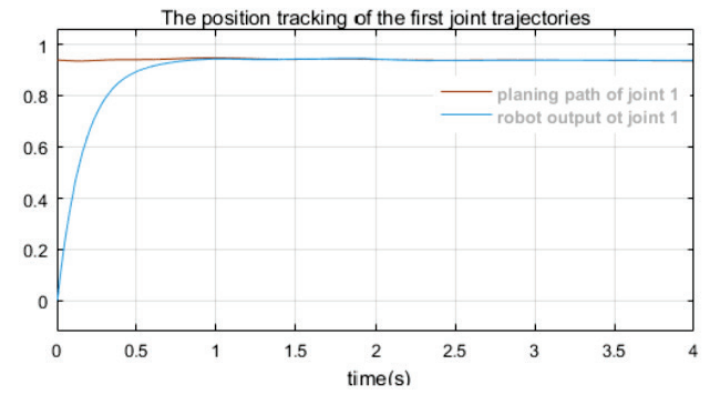

Figure 14 The position tracking of the first joint trajectories

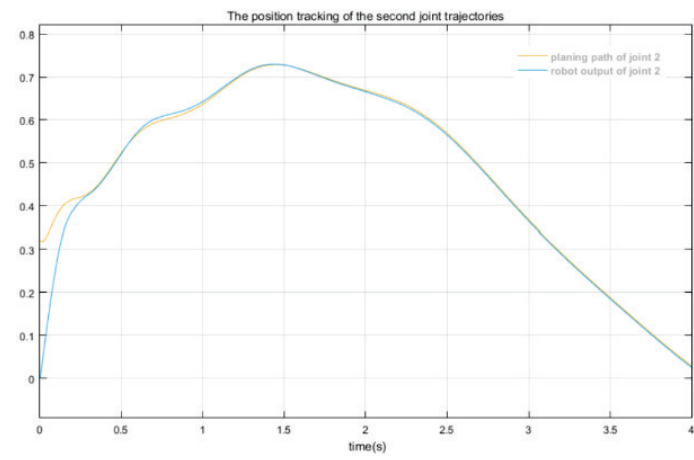

Figure 15 The position tracking of the second joint trajectories

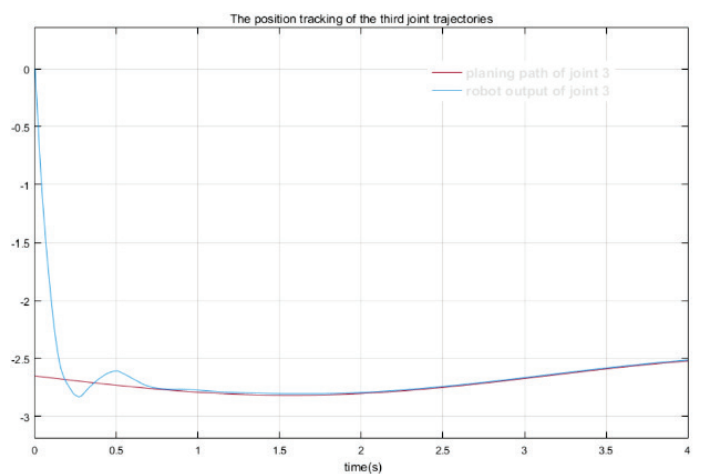

Figure 16 The position tracking of the third joint trajectories

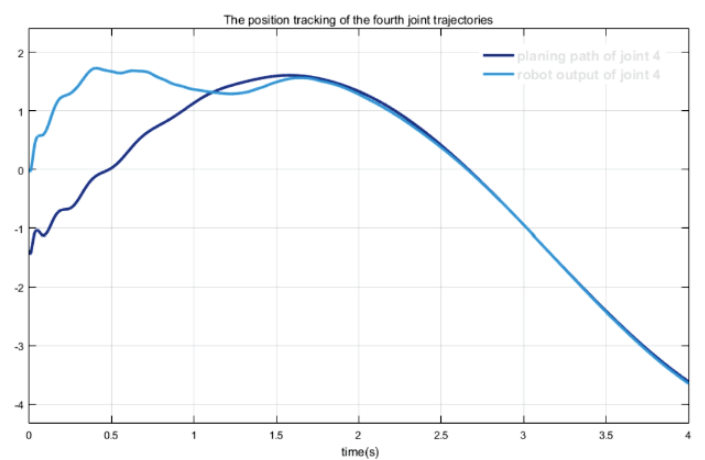

Figure 17 The position tracking of the fourth joint trajectories

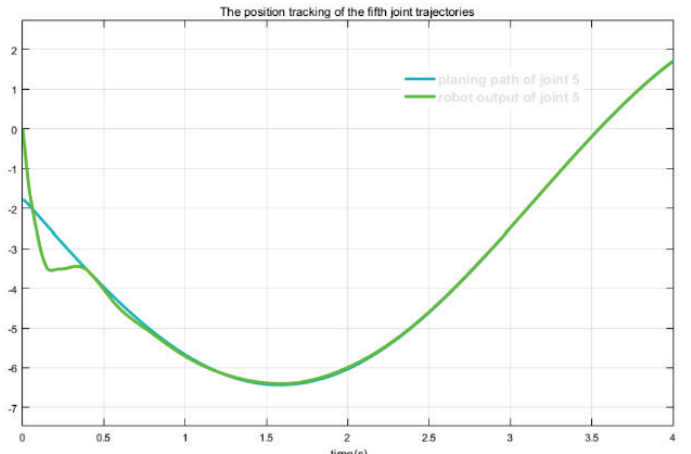

Figure 18 The position tracking of the fifth joint trajectories

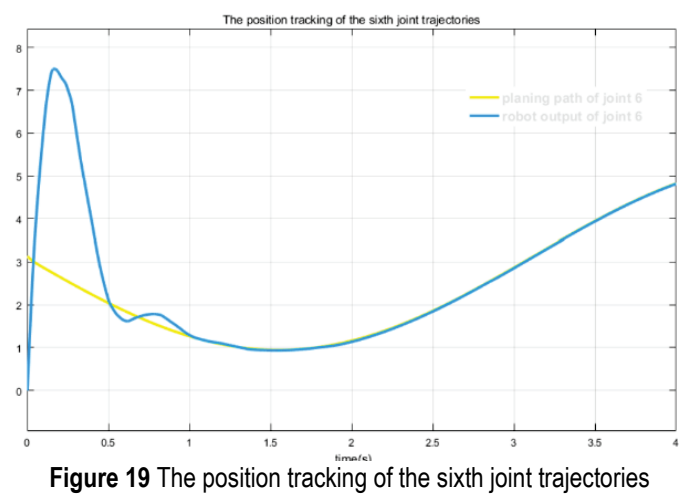

In the controlling process, there may be infinite solutions or no solutions when inverse displacements get from computed through the posture calculated by robotic system with singular posture points. We add output threshold limited module after URBF controller to limit the maximum and minimum. Fig. 20 shows the output error of each joint whose threshold is set to 200 (the dotted line is the singular point area in the figure), and Fig. 12 shows the output error of each joint whose threshold is set to 20 (the dotted line is singular point in the figure). From two figures, it can be seen that UBRF meets the requirements and can effectively deal with the inaccurate problem which is from calculating of singular points.

There are two advantages for the control system: Firstly, two different controllers will be synchronized through a synchronous time step acquired from path planning time step. Secondly, the URBF neural network can compensate for the CTL control system.

In surgical operations, the efficiency of the path planning process that includes an ILC structure could be improved as the iteration time is increased. Path planning process will be finished in a very short time, and then, the robot control could be performed by doctors.

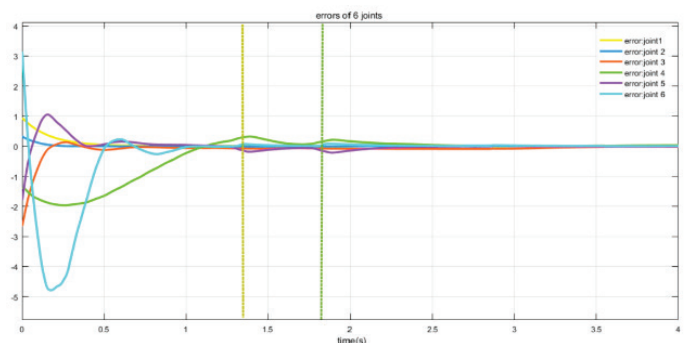

Figure 20 The output error of each joint with the threshold set to 200

Table 3 The 6 joints locations for the robot and the puncture actuator at the final

\begin{tabular}{|c|c|}
\multicolumn{2}{c}{ time step } \\
\hline Actuator & 0.05403 \\
\hline \multirow{4}{*}{ Robot joint } & 0.9358 \\
\cline { 2 - 2 } & 0.02861 \\
\cline { 2 - 2 } & -2.522 \\
\cline { 2 - 2 } & -3.708 \\
\cline { 2 - 2 } & 1.747 \\
\cline { 2 - 2 } & \\
\cline { 2 - 2 } & \\
\hline
\end{tabular}

Table 4 The expectation locations of the robotic joints and the puncture actuator

\begin{tabular}{|l|c|}
\hline \multicolumn{2}{|c}{ at the final time step } \\
\hline Actuator & 0.0619 \\
\hline \multirow{4}{*}{ Robot joint } & 0.9359 \\
\cline { 2 - 2 } & 0.0287 \\
\cline { 2 - 2 } & -2.522 \\
\cline { 2 - 2 } & -3.614 \\
\cline { 2 - 2 } & 1.701 \\
\cline { 2 - 2 } & \\
\end{tabular}


Table 5 The robot joints Errors and the actuator error at the last time step

\begin{tabular}{|c|c|}
\hline Actuator & -0.007873 \\
\hline \multirow{4}{*}{ Robot joint } & -0.001739 \\
\cline { 2 - 2 } & 0.003293 \\
\cline { 2 - 2 } & -0.008526 \\
\cline { 2 - 2 } & 0.03216 \\
\cline { 2 - 2 } & -0.006386 \\
\cline { 2 - 2 } & 0.009588 \\
\hline
\end{tabular}

\section{CONCLUSIONS}

In this study, a URBF compensating controller is proposed for the computed torque control system. It is proved that the output precision of the 7-DOF surgical robot is sufficient for the surgical technical requirements.

It is demonstrated through simulations that both channels, both the robotic arm and the puncture actuator, can execute the predefined path planning, successfully.

AURBF compensating controller is adopted in this control system. The controller settings are adjusted in accordance with the CTL control loop of the robot.

The merging process of the direction and the position for two mentioned channels could be realized through inserting the needle in accordance with the synchronizing time step. Thus, it can effectively improve the puncture robot performance.

During the surgery, an automatic and on-line supervised puncture process is performed. The online supervision based automatic needle inserting is a new percutaneous puncture robotic system.

In this paper, a new control approach for the automatic percutaneous puncture robot is proposed. In the future, this control approach should be applied to the actual 7-axis robot. Further test is dispensable for performance evaluation of the proposed control approach.

\section{REFERENCES}

[1] Zarrouk, Z., Chemorit, A., \& Poignet, P. (2013). Force feedback control for compensation of physiological motions in beating heart surgery with real-time experiments. $3^{\text {rd }}$ International Conference on Systems and Control, Algiers, 2013, pp. 956-961. https://doi.org/10.1109/ICoSC.2013.6750972

[2] Cagneau, B., Zemiti, N., Bellot, D. et al. (2007). Physiological Motion Compensation in Robotized Surgery using Force Feedback Control. IEEE International Conference on Robotics and Automation, 1881-1886. https://doi.org/10.1109/ROBOT.2007.363596

[3] Kobayashi, Y., Moreira, P., Liu, C. et al. (2011). Haptic feedback control in medical robots through fractional viscoelastic tissue model. Conf Proc IEEE Eng Med Biol Soc, (4), 6704-6708. https://doi.org/10.1109/IEMBS.2011.6091653

[4] Qu, J., Li, J., Zhang, L. et al. (2013). Design of a novel forcereflecting haptic device for minimally invasive surgery robot. ICME International Conference on Complex Medical Engineering, 357-362.

[5] Alexandre, B., Zhou, J., Yang, G. et al. (2009). Motion Compensated Tomography Reconstruction of Coronary Arteries in Rotational Angiography. IEEE transactions on bio-medical engineering, 56(4), 1254-7. https://doi.org/10.1109/TBME.2008.2005205

[6] Danilo, B., Calinon, S., \& Caldwell, D. G. (2017). Learning autonomous behaviours for the body of a flexible surgical robot. Kluwer Academic Publishers, 2017.

[7] Cuong, P. \& Nan, W. Y. (2016). Adaptive trajectory tracking neural network control with robust compensator for robot manipulators. Neural Computing \& Applications, 27(2), 525536. https://doi.org/10.1007/s00521-015-1873-4

[8] Jankovics, V., Mátéfi-Tempfli, S., \& Manoonpong, P. (2016) Artificial Neural Network Based Compliant Control for Robot Arms. International Conference on Simulation of Adaptive Behavior, 91-100. https://doi.org/10.1007/978-3-319-43488-9_9

[9] Lu, B. \& Pu, J. (2018). Sliding mode control based on fuzzy switching gain for the robot arm. Chinese Automation Congress. https://doi.org/10.1109/CAC.2017.8243178

[10] Sisbot, E. A., Marin-Urias, L. F., Alami, R. et al. (2007). A Human Aware Mobile Robot Motion Planner. IEEE Transactions on Robotics, 23(5), 874-883. https://doi.org/10.1109/TRO.2007.904911

[11] Lewis, F. L., Yegildirek, A., \& Liu, K. (1996). Multilayer neural-net robot controller with guaranteed tracking performance. IEEE Press. https://doi.org/10.1109/72.485674

[12] Lewis, F. L., Yesildirak, A., \& Jagannathan, S. (1998). Neural Network Control of Robot Manipulators and Nonlinear Systems. Taylor \& Francis, Inc.

[13] Tang, L. \& Liu, Y. J. (2014). Adaptive neural network control of robot manipulator using reinforcement learning. Journal of Vibration \& Control, 20(14), 2162-2171. https://doi.org/10.1177/1077546313482171

[14] Haddadin, S., Albu-SchäFfer, A., De Luca, A. et al. (2008). Collision detection and reaction: A contribution to safe physical Human-Robot Interaction. IEEE/rsj International Conference on Intelligent Robots and Systems, 3356-3363. https://doi.org/10.1109//ROS.2008.4650764

[15] Luca, A. D., Albu-Schaffer, A., Haddadin, S. et al. (2007). Collision Detection and Safe Reaction with the DLR-III Lightweight Manipulator Arm. IEEE/rsj International Conference on Intelligent Robots and Systems, 1623-1630.

[16] Jiang, M., Fan, M. Q., Li, A. M. et al. (2016). Coordination control of dual-arm robot based on modeled predictive control IEEE International Conference on Real-Time Computing and Robotics, 495-499. https://doi.org/10.1109/RCAR.2016.7784079

[17] Wilson, J., Charest, M., \& Dubay, R. (2016). Non-linear model predictive control schemes with application on a 2 link vertical robot manipulator. Robotics \& Computer Integrated Manufacturing, 41(C), 23-30. https://doi.org/10.1016/j.rcim.2016.02.003

[18] Precup, R. E., Radac, M. B., Roman, R. C. et al. (2017). Model-free sliding mode control of nonlinear systems: Algorithms and experiments. Information Sciences, 381, 176192. https://doi.org/10.1016/j.ins.2016.11.026

[19] Spall, J. C. \& Cristion, J. A. (1998). Model-free control of nonlinear stochastic systems with discrete-time measurements. Automatic Control IEEE Transactions on, 43(9), 1198-1210. https://doi.org/10.1109/9.718605

[20] Reham, H., Bendary, F., \& Elserafi, K. (2016). Trajectory Tracking Control for Robot Manipulator using Fractional Order-Fuzzy-PID Controller. International Journal of Computer Applications, 134(15), 22-29. https://doi.org/10.5120/ijca2016908155

[21] Naik, P. R., Samantaray, J., Roy, B. K. et al. (2016). 2-DOF robot manipulator control using fuzzy PD control with SimMechanics and sliding mode control: A comparative study. International Conference on Energy, Power and Environment: Towards Sustainable Growth, 1-6. https://doi.org/10.1109/EPETSG.2015.7510101

[22] Abdelhamid, B., Mouhamed, C., \& Najib, E. (2017). Indirect Robust Adaptive Fuzzy Control of Uncertain Two Link Robot Manipulator. https://doi.org/10.1007/978-3-319-48929-2_10

[23] Baek, J., Jin, M., \& Han, S. (2016). A New Adaptive SlidingMode Control Scheme for Application to Robot Manipulators. IEEE Transactions on Industrial Electronics, 63(6), 36283637. https://doi.org/10.1109/TIE.2016.2522386 
[24] Rossomando, F. G. \& Sori, C. M. (2016). Adaptive Neural Sliding Mode Control in Discrete Time for a SCARA robot arm. IEEE Latin America Transactions, 14(6), 2556-2564. https://doi.org/10.1109/TLA.2016.7555218

[25] Tonietti, G., Schiavi, R., \& Bicchi, A. (2006). Design and Control of a Variable Stiffness Actuator for Safe and Fast Physical Human/Robot Interaction. IEEE International Conference on Robotics and Automation, 526-531.

[26] Bicchi, A., Tonietti, G., Bavaro, M. et al. (2005). Variable Stiffness Actuators for Fast and Safe Motion Control. Springer Tracts in Advanced Robotics, 15, 527-536. https://doi.org/10.1007/11008941_56

[27] Danilo, B., Calinon, S., \& Caldwell, D. G. (2017). Learning autonomous behaviours for the body of a flexible surgical robot. Kluwer Academic Publishers.

[28] Patrice, L. \& Just, L. H. A 7-DOF redundantly actuated parallel haptic device combining 6-DOF manipulation and 1DOF grasping. Mechanism and Machine Theory, 134, 349-36. https://doi.org/10.1016/j.mechmachtheory.2019.01.002

[29] Çalişkan, A. \& Çevik, U. (2018). An Efficient Noisy Pixels Detection Model for CT Images using Extreme Learning Machines. Tehnicki vjesnik - Technical Gazette, 25(3). https://doi.org/10.17559/TV-20171220221947

\section{Contact information:}

\section{Liandong WANG}

(Corresponding author)

School of Mechanical and Aerospace Engineering (SAME),

Jilin University, No. 2699 Qianjin Street, Changchun, 130012 China

E-mail: wangliandong_jlu@163.com

\section{Xiaoqin ZHOU}

School of Mechanical and Aerospace Engineering (SAME),

Jilin University, No. 2699 Qianjin Street, Changchun, 130012 China

\section{Tiehua HU}

China Academy of Machinery Science \& Technology (CAM),

No. 2 Shouti South Road, Haidian District, Beijing, 100044 China 